\title{
Three positive periodic solutions of second order nonlinear neutral functional differential equations with delayed derivative
}

\section{He Yang ${ }^{1 *}$ and Lu Zhang ${ }^{1}$}

"Correspondence:

yanghe256@163.com

${ }^{1}$ College of Mathematics and

Statistics, Northwest Normal

University, Lanzhou, China

\section{Springer}

\begin{abstract}
This paper deals with the existence of three positive periodic solutions for a class of second order neutral functional differential equations involving the delayed derivative term in nonlinearity

$(x(t)-c x(t-\delta))^{\prime \prime}+a(t) g(x(t)) x(t)=\lambda b(t) f\left(t, x(t), x\left(t-\tau_{1}(t)\right), x^{\prime}\left(t-\tau_{2}(t)\right)\right)$. By utilizing the perturbation method of positive operator and Leggett-Williams fixed point theorem, a group of sufficient conditions are established.
\end{abstract}

MSC: $34 \mathrm{~B} 18 ; 34 \mathrm{C} 25$

Keywords: Neutral functional differential equation; Positive periodic solution; Delayed derivative; Cone; Leggett-Williams fixed point theorem

\section{Introduction}

In the present work, we study the existence of three positive periodic solutions for the second order neutral functional differential equation of the form

$$
(x(t)-c x(t-\delta))^{\prime \prime}+a(t) g(x(t)) x(t)=\lambda b(t) f\left(t, x(t), x\left(t-\tau_{1}(t)\right), x^{\prime}\left(t-\tau_{2}(t)\right)\right),
$$

where $\lambda>0$ is a positive parameter, $c, \delta$ are constants, and $|c|<1 . a(t), b(t)$ are nonnegative $\omega$-periodic continuous functions, $\tau_{i}(t), i=1,2$, are continuous $\omega$-periodic functions, $f$ : $\mathbb{R} \times[0,+\infty)^{2} \times \mathbb{R} \rightarrow[0,+\infty)$ is a continuous function, and $f(t, u, v, w)$ is $\omega$-periodic with respect to $t, g \in C([0,+\infty),[0,+\infty))$.

Neutral functional differential equations have a wide range of applications in the field of physics, biology, economics, and so on, see [1-14] for more details. In [15], the authors pointed out that the growth of single or multiple species was mainly affected by seasonal changes (especially cyclical changes) and time lags. So it is important to study the periodic solutions of such models. The issues of the existence of positive periodic solutions of neutral functional differential equations have received more attention in recent years, see [7-14]. The existence of positive periodic solutions for first order neutral functional differential equations has been studied by many authors, see [7-12] and the references therein. But the research results on the case of second order are more seldom.

(c) The Author(s) 2020. This article is licensed under a Creative Commons Attribution 4.0 International License, which permits use, sharing, adaptation, distribution and reproduction in any medium or format, as long as you give appropriate credit to the original author(s) and the source, provide a link to the Creative Commons licence, and indicate if changes were made. The images or other third party material in this article are included in the article's Creative Commons licence, unless indicated otherwise in a credit line to the material. If material is not included in the article's Creative Commons licence and your intended use is not permitted by statutory regulation or exceeds the permitted use, you will need to obtain permission directly from the copyright holder. To view a copy of this licence, visit http://creativecommons.org/licenses/by/4.0/. 
In [13], the authors studied the existence, multiplicity, and nonexistence of positive periodic solutions of second order neutral functional differential equations of the form

$$
(x(t)-c x(t-\delta))^{\prime \prime}+a(t) x(t)=\lambda b(t) f(x(t-\tau(t))),
$$

where $\lambda>0$ is a positive parameter, $c, \delta$ are constants, and $|c|<1, a(t), b(t)$ are nonnegative $\omega$-periodic continuous functions. But the nonlinear term does not contain the derivative term.

Recently, Li [14] discussed the existence and nonexistence of positive $\omega$-periodic solutions of second order neutral functional differential equations with delayed derivative in nonlinear term by using the positive operator perturbation method and the fixed point index theory

$$
(x(t)-c x(t-\delta))^{\prime \prime}+a(t) x(t)=f\left(t, x(t), x(t-\tau(t)), x^{\prime}(t-\gamma(t))\right),
$$

where $\delta>0,|c|<1, a \in C(\mathbb{R}, \infty)$ is an $\omega$ periodic function, $f: \mathbb{R} \times[0, \infty)^{2} \times \mathbb{R} \rightarrow[0, \infty)$ is continuous, and $f(t, u, v, w)$ is $\omega$-periodic with respect to $t, \tau, \gamma \in C(\mathbb{R},[0, \infty))$ are $\omega$ periodic functions. But he did not consider the multiplicity of the positive periodic solutions.

Motivated by the above mentioned results, in this work, by using a different method, we mainly study the existence and multiplicity of positive periodic solutions for a class of second order neutral nonlinear functional differential equations with delayed derivative of the form (1).

Let $C_{\omega}(\mathbb{R})$ be the Banach space of all continuous $\omega$-periodic functions endowed with the norm $\|x\|_{C}=\max _{t \in[0, \omega]}|x(t)|, C_{\omega}^{1}(\mathbb{R})$ be the Banach space of all continuous differentiable $\omega$-periodic functions with the norm $\|x\|_{C^{1}}=\|x\|_{C}+\left\|x^{\prime}\right\|_{C}$. In general, for $n \in \mathbb{N}, C_{\omega}^{n}(\mathbb{R})$ represents the Banach space of all $n$th order continuous differentiable $\omega$-periodic functions. Let $C_{\omega}^{+}(\mathbb{R})=C_{\omega}(\mathbb{R},[0, \infty))$ be a nonnegative function cone in $C_{\omega}(\mathbb{R})$.

The main results of the present paper are summarized as follows:

(i) We establish the existence (and uniqueness) of $\omega$-periodic solutions for the corresponding linear second order neutral functional differential equation

$$
(x(t)-c x(t-\delta))^{\prime \prime}+a(t) g(x(t)) x(t)=\lambda h(t), \quad t \in \mathbb{R} .
$$

See Lemma 5.

(ii) We provide the strong positive estimate and $C^{1}$-estimate of the periodic solution operator by using the positive operator perturbation method, see Lemma 6 .

(iii) Let

$$
K=\left\{x \in C_{\omega}^{1}(\mathbb{R}): x(t) \geq \sigma\|x\|_{C},\left|x^{\prime}(\tau)\right| \leq C_{0}|x(t)|, \tau, t \in \mathbb{R}\right\},
$$

where $\sigma$ and $C_{0}$ will be specified later. We define an operator $Q_{\lambda}$ which maps $K$ into itself and prove that the operator $Q_{\lambda}$ has at least three positive fixed points by using Leggett-Williams fixed point theorem, see Theorem 1.

In this paper, we always assume that

(H1) $f \in C\left(\mathbb{R} \times[0, \infty)^{2} \times \mathbb{R},[0, \infty)\right), f(t, u, v, w)$ is nondecreasing with respect to $u, v, w$ and $\omega$-periodic in $t ; g \in C([0, \infty),[0, \infty))$; 
$(H 2) \quad a, b \in C_{\omega}^{+}(\mathbb{R}), \bar{b}:=\frac{1}{\omega} \int_{0}^{\omega} b(s) d s>0$, and $\tau_{i} \in C_{\omega}(\mathbb{R}), i=1,2$;

(H3) there exist two positive constants $d$ and $D$ satisfying $0<d \leq a(t) g(x(t)) \leq D<\left(\frac{\pi}{\omega}\right)^{2}$ for any $t \in[0, \omega], x \in C_{\omega}^{+}(\mathbb{R})$.

\section{Preliminaries}

Firstly, let $0<M<\left(\frac{\pi}{\omega}\right)^{2}$. We consider the second order linear ordinary differential equation

$$
x^{\prime \prime}(t)+M x(t)=\lambda h(t), \quad h \in C_{\omega}(\mathbb{R}) .
$$

By Lemma 2.1 of [14], the following lemma is obtained.

Lemma 1 For $\forall h \in C_{\omega}(\mathbb{R})$, linear equation (2) has a unique $\omega$-periodic solution $x \in C_{\omega}^{2}(\mathbb{R})$ expressed by

$$
x(t)=\lambda \int_{t-\omega}^{t} U(t-s) h(s) d s:=T_{\lambda}(h)(t), \quad t \in \mathbb{R},
$$

where

$$
U(t)=\frac{\cos \sqrt{M}\left(t-\frac{\omega}{2}\right)}{2 \sqrt{M} \sin \frac{\sqrt{M} \omega}{2}}, \quad 0 \leq t \leq \omega .
$$

And the operator $T_{\lambda}: C_{\omega}(\mathbb{R}) \rightarrow C_{\omega}^{1}(\mathbb{R})$ is a linear completely continuous operator.

For the sake of brevity, let $\beta=\sqrt{M}$ and denote

$$
\begin{aligned}
& L=\max _{t \in[0, \omega]} U(t)=\frac{1}{2 \beta \sin \frac{\beta \omega}{2}}, \quad l=\min _{t \in[0, \omega]} U(t)=\frac{\cos \frac{\beta \omega}{2}}{2 \beta \sin \frac{\beta \omega}{2}}, \\
& L_{1}=\max _{t \in[0, \omega]}\left|U^{\prime}(t)\right|=\max _{t \in[0, \omega]} \frac{\left|\sin \beta\left(t-\frac{\omega}{2}\right)\right|}{2 \beta \sin \frac{\beta \omega}{2}}=\frac{1}{2}, \\
& \sigma=\frac{l}{L}=\cos \frac{\beta \omega}{2}, \quad C_{0}=\frac{L_{1}}{l}=\beta \tan \frac{\beta \omega}{2} .
\end{aligned}
$$

Then

$$
0<\sigma<1, \quad 0<l \leq U(t) \leq L .
$$

Clearly, if $h \in C_{\omega}^{+}(\mathbb{R})$, the solution $x \in C_{\omega}^{2}(\mathbb{R})$ of (2) is positive.

Define a cone $K$ in $C_{\omega}^{1}(\mathbb{R})$ by

$$
K=\left\{x \in C_{\omega}^{1}(\mathbb{R}): x(t) \geq \sigma\|x\|_{C},\left|x^{\prime}(\tau)\right| \leq C_{0}|x(t)|, \tau, t \in \mathbb{R}\right\} .
$$

Lemma $2 T_{\lambda}\left(C_{\omega}^{+}(\mathbb{R})\right) \subset K$ and $\left\|T_{\lambda}\right\| \leq \frac{\lambda}{M}$.

Proof Let $h \in C_{\omega}^{+}(\mathbb{R})$. By (3) and (4), we have

$$
x(t)=\lambda \int_{t-\omega}^{t} U(t-s) h(s) d s \leq \lambda L \int_{t-\omega}^{t} h(s) d s=\lambda L \int_{0}^{\omega} h(s) d s, \quad \forall t \in \mathbb{R} .
$$


That is, $\|x\|_{C} \leq \lambda L \int_{0}^{\omega} h(s) d s$. Hence we have

$$
x(t) \geq \lambda l \int_{t-\omega}^{t} h(s) d s=\lambda l \int_{0}^{\omega} h(s) d s \geq \sigma\|x\|_{C}, \quad \forall t \in \mathbb{R} .
$$

For $\forall \tau \in \mathbb{R}$, noticing that $x^{\prime}(\tau)=\lambda \int_{\tau-\omega}^{\tau} U^{\prime}(\tau-s) h(s) d s$, we have

$$
\begin{aligned}
\left|x^{\prime}(\tau)\right| & \leq \lambda \int_{\tau-\omega}^{\tau}\left|U^{\prime}(\tau-s)\right| h(s) d s \\
& \leq \lambda L_{1} \int_{\tau-\omega}^{\tau} h(s) d s \\
& =\lambda L_{1} \int_{0}^{\omega} h(s) d s \\
& \leq C_{0} x(t) .
\end{aligned}
$$

Consequently, by (5), $T_{\lambda}\left(C_{\omega}^{+}(\mathbb{R})\right) \subset K$. In addition, for $h \in C_{\omega}^{+}(\mathbb{R})$, the inequality

$$
\left|T_{\lambda} h(t)\right| \leq \lambda \int_{t-\omega}^{t} U(t-s) d s\|h\|_{C}=\frac{\lambda}{M}\|h\|_{C}
$$

implies that $\left\|T_{\lambda}\right\| \leq \frac{\lambda}{M}$ and the proof is complete.

In order to prove the existence of $\omega$-periodic solutions of equation (1), we consider the corresponding linear neutral functional differential equation

$$
(x(t)-c x(t-\delta))^{\prime \prime}+a(t) g(x(t)) x(t)=\lambda h(t), \quad h \in C_{\omega}(\mathbb{R}) .
$$

Define a linear operator $A: C_{\omega}(\mathbb{R}) \rightarrow C_{\omega}(\mathbb{R})$ by

$$
A x(t)=x(t)-c x(t-\delta), \quad t \in \mathbb{R}, x \in C_{\omega}(\mathbb{R}) .
$$

Then $A: C_{\omega}(\mathbb{R}) \rightarrow C_{\omega}(\mathbb{R})$ is bounded.

Lemma $3([11,12,14])$ If $|c| \neq 1$, then the operator $A$, defined by (7), has a linear bounded inverse operator $A^{-1}$ on $C_{\omega}(\mathbb{R})$ given by

$$
\left(A^{-1} y\right)(t)= \begin{cases}\sum_{j \geq 0} c^{j} y(t-j \delta), & |c|<1 \\ -\sum_{j \geq 1} c^{-j} y(t+j \delta), & |c|>1\end{cases}
$$

and

$$
\left\|A^{-1} y\right\|_{C} \leq \frac{\|y\|_{C}}{|1-| c||}
$$

Lemma 4 If $|c|<\sigma$, then for any $y \in K$, we have

$$
A^{-1} y(t) \geq \frac{\sigma-|c|}{1-c^{2}}\|y\|_{C}, \quad \forall t \in \mathbb{R}
$$


Proof For any $y \in K$, by virtue of Lemma 3, we have

$$
\begin{aligned}
A^{-1} y(t) & =\sum_{j \geq 0} c^{j} y(t-j \delta)=\sum_{j \geq 0}|c|^{2 j} y(t-2 j \delta)-\sum_{j \geq 1}|c|^{2 j-1} y(t-(2 j-1) \delta) \\
& \geq \sum_{j \geq 0}|c|^{2 j} \sigma\|y\|_{C}-\sum_{j \geq 1}|c|^{2 j-1}\|y\|_{C} \\
& \geq\left(\frac{\sigma-|c|}{1-c^{2}}\right)\|y\|_{C} .
\end{aligned}
$$

Then the proof of Lemma 4 is complete.

Let $y=A x$. Then by (7), equation (6) can be rewritten as

$$
y^{\prime \prime}+a(t) g\left(\left(A^{-1} y\right)(t)\right)\left(A^{-1} y\right)(t)=\lambda h(t), \quad t \in \mathbb{R} .
$$

It is available from Lemma 3 that when $y \in C_{\omega}^{1}(\mathbb{R}), A^{-1} y \in C_{\omega}^{1}(\mathbb{R})$, and $\left(A^{-1} y\right)^{\prime}=A^{-1} y^{\prime}$, when $y \in C_{\omega}^{2}(\mathbb{R}), A^{-1} y \in C_{\omega}^{2}(\mathbb{R})$, and $\left(A^{-1} y\right)^{\prime \prime}=A^{-1} y^{\prime \prime}$. Therefore, $x \in C_{\omega}^{2}(\mathbb{R})$ is an $\omega$-periodic solution of equation (6) if and only if $y=A x \in C_{\omega}^{2}(\mathbb{R})$ is an $\omega$-periodic solution of equation (8).

Lemma 5 If $|c|<\frac{d}{D+d}$, equation (8) has a unique $\omega$-periodic solution $y \in C_{\omega}^{2}(\mathbb{R})$ for any $h \in C_{\omega}(\mathbb{R})$. When $h \in C_{\omega}^{+}(\mathbb{R})$ and $|c|<\min \left\{\frac{d}{D+d}, \frac{\sigma(M-D)}{\sigma(M-D)+D}\right\}$, the $\omega$-periodic solution $y \in K$.

Proof Define an operator $B_{\lambda}: C_{\omega}(\mathbb{R}) \rightarrow C_{\omega}(\mathbb{R})$ by

$$
B_{\lambda} y(t)=\frac{1}{\lambda}\left[\left(M-a(t) g\left(\left(A^{-1} y\right)(t)\right)\right) y(t)-G(y(t))\right], \quad t \in \mathbb{R}
$$

where $G(y(t))=c a(t) g\left(\left(A^{-1} y\right)(t)\right)\left(A^{-1} y\right)(t-\delta)$. Then equation (8) can be rewritten as

$$
y^{\prime \prime}(t)+M y(t)=\lambda B_{\lambda} y(t)+\lambda h(t), \quad t \in \mathbb{R} .
$$

By Lemma 1, we have

$$
\left(I-T_{\lambda} B_{\lambda}\right) y(t)=T_{\lambda} h(t), \quad t \in \mathbb{R} .
$$

It follows from (9) that

$$
\begin{aligned}
\left|B_{\lambda} y(t)\right| & =\left|\frac{1}{\lambda}\left[\left(M-a(t) g\left(\left(A^{-1} y\right)(t)\right)\right) y(t)-c a(t) g\left(\left(A^{-1} y\right)(t)\right)\left(A^{-1} y\right)(t-\delta)\right]\right| \\
& \leq \frac{1}{\lambda}\left[(M-d)\|y\|_{C}+|c| D \frac{\|y\|_{C}}{1-|c|}\right] \\
& =\frac{1}{\lambda}\left(M-d+\frac{|c| D}{1-|c|}\right)\|y\|_{C} .
\end{aligned}
$$

Consequently,

$$
\left\|B_{\lambda}\right\| \leq \frac{1}{\lambda}\left(M-d+\frac{|c| D}{1-|c|}\right)
$$


Combining this fact with $\left\|T_{\lambda}\right\| \leq \frac{\lambda}{M}$, we have

$$
\left\|T_{\lambda} B_{\lambda}\right\| \leq\left\|T_{\lambda}\right\|\left\|B_{\lambda}\right\| \leq \frac{1}{M}\left(M-d+\frac{|c| D}{1-|c|}\right) .
$$

Then $\left\|T_{\lambda} B_{\lambda}\right\|<1$ because of $|c|<\frac{d}{D+d}$. Hence the operator $I-T_{\lambda} B_{\lambda}$ has a bounded inverse operator $\left(I-T_{\lambda} B_{\lambda}\right)^{-1}$ which can be expressed by

$$
\left(I-T_{\lambda} B_{\lambda}\right)^{-1}=\sum_{n=0}^{\infty}\left(T_{\lambda} B_{\lambda}\right)^{n}
$$

Therefore, operator equation (11) has a unique $\omega$-periodic solution $y \in C_{\omega}^{2}(\mathbb{R})$ expressed by

$$
y=\left(I-T_{\lambda} B_{\lambda}\right)^{-1} T_{\lambda} h=\sum_{n=0}^{\infty}\left(T_{\lambda} B_{\lambda}\right)^{n} T_{\lambda} h
$$

Let $z=T_{\lambda} h$ for any $h \in C_{\omega}^{+}(\mathbb{R})$. By Lemma 2 , we get $z \in K$. Then we have

$$
\begin{aligned}
B_{\lambda} z(t) & =\frac{1}{\lambda}\left[\left(M-a(t) g\left(\left(A^{-1} z\right)(t)\right)\right) z(t)-c a(t) g\left(\left(A^{-1} z\right)(t)\right)\left(A^{-1} z\right)(t-\delta)\right] \\
& \geq \frac{1}{\lambda}\left[(M-D) z(t)-|c| D\left\|A^{-1} z\right\|_{C}\right] \\
& \geq \frac{1}{\lambda}\left[(M-D) \sigma\|z\|_{C}-\frac{|c| D}{1-|c|}\|z\|_{C}\right] \\
& =\frac{1}{\lambda}\left[(M-D) \sigma-\frac{|c| D}{1-|c|}\right]\|z\|_{C} .
\end{aligned}
$$

Since $|c|<\frac{\sigma(M-D)}{\sigma(M-D)+D}$, it follows that

$$
(M-D) \sigma-\frac{|c| D}{1-|c|}>0
$$

Hence $B_{\lambda} z(t) \geq 0$ for any $t \in \mathbb{R}$, that is, $B_{\lambda} z \in C_{\omega}^{+}(\mathbb{R})$. Then applying Lemma 2 again, $\left(T_{\lambda} B_{\lambda}\right) z=T_{\lambda} B_{\lambda} z \in K$. Consequently, $\left(T_{\lambda} B_{\lambda}\right)^{n} z \in K$ for $\forall n \in \mathbb{N}$. By boundedness of the linear operator $T_{\lambda}: C_{\omega}(\mathbb{R}) \rightarrow C_{\omega}^{1}(\mathbb{R}), \sum_{n=1}^{\infty}\left(T_{\lambda} B_{\lambda}\right)^{n} z$ is convergence in $C_{\omega}^{1}(\mathbb{R})$. Since the cone $K \subset C_{\omega}^{1}(\mathbb{R})$ is closed, then by (12), we have

$$
y=\sum_{n=0}^{\infty}\left(T_{\lambda} B_{\lambda}\right)^{n} T_{\lambda} h=\sum_{n=0}^{\infty}\left(T_{\lambda} B_{\lambda}\right)^{n} z \in K .
$$

This completes the proof of Lemma 5.

Lemma 6 Let $h \in C_{\omega}^{+}(\mathbb{R})$ and $|c|<\min \left\{\frac{d}{D+d}, \frac{\sigma(M-D)}{\sigma(M-D)+D}\right\}$. Then $h^{*}(\cdot):=b(\cdot) h(\cdot)$ belongs to $C_{\omega}^{+}(\mathbb{R})$, and the operator $S_{\lambda}: C_{\omega}^{+}(\mathbb{R}) \rightarrow C_{\omega}^{+}(\mathbb{R})$ defined by

$$
\left(S_{\lambda} h^{*}\right)(t)=\int_{t-\omega}^{t} U(t-s)\left[\left(M-a(s) g\left(A^{-1} y\right)(s)\right) y(s)-G(y(s))+\lambda h^{*}(s)\right] d s
$$

maps $C_{\omega}^{+}(\mathbb{R})$ to $K$ and it is completely continuous. 
Proof By Lemma 1, $y=S_{\lambda} h^{*} \in C_{\omega}^{2}(\mathbb{R})$ is an $\omega$-periodic solution of equation (10) since equation (10) is equivalent to equation (8). By Lemma 5, equation (8) has a unique $\omega$-periodic solution $y \in C_{\omega}^{2}(\mathbb{R})$ expressed by

$$
y=\sum_{n=0}^{\infty}\left(T_{\lambda} B_{\lambda}\right)^{n} T_{\lambda} h^{*} .
$$

By (13), we know that $y \in K$. Hence $S_{\lambda}: C_{\omega}^{+}(\mathbb{R}) \rightarrow K$ and it is completely continuous. This completes the proof of Lemma 6.

At the end of this section, we introduce a fixed point theorem, which will be used in the proof of our main result.

Let $(X,\|\cdot\|)$ be a real Banach space and $K$ be a cone in $X$. A map $\rho$ is called a nonnegative continuous concave function on $K$ if $\rho: K \rightarrow[0,+\infty)$ is continuous and

$$
\rho(t x+(1-t) y) \geq t \rho(x)+(1-t) \rho(y)
$$

for all $x, y \in K$ and $t \in[0,1]$.

Let $0<r<R$ and $\rho$ be a nonnegative continuous concave function on $K$, set

$$
\begin{aligned}
& K_{r}=\{x \in K:\|x\|<r\}, \quad \bar{K}_{r}=\{x \in K:\|x\| \leq r\}, \\
& K(\rho, r, R)=\{x \in K: r \leq \rho(x),\|x\| \leq R\} .
\end{aligned}
$$

Lemma $7([16,17])$ Let $Q: \bar{K}_{R} \rightarrow \bar{K}_{R}$ be a completely continuous mapping and $\rho$ be a nonnegative continuous concave function on $K$ with $\rho(x) \leq\|x\|$ for all $x \in \bar{K}_{R}$. Suppose that there exist positive constants $r, r_{1}, r_{2}, R$ with $0<r<r_{1}<r_{2}<R$ such that

(a) $\left\{x \in K\left(\rho, r_{1}, r_{2}\right): \rho(x)>r_{1}\right\} \neq \emptyset$ and $\rho(Q x)>r_{1}$ for $x \in K\left(\rho, r_{1}, r_{2}\right)$;

(b) $\|Q x\|<r$ for $x \in \bar{K}_{r}$;

(c) $\rho(Q x)>r_{1}$ for $x \in K\left(\rho, r_{1}, R\right)$ with $\|Q x\|>r_{2}$.

Then $Q$ has at least three fixed points $x_{1}, x_{2}, x_{3}$ satisfying

$$
x_{1} \in K_{r}, x_{2} \in\left\{x \in K\left(\rho, r_{1}, R\right): \rho(x)>r_{1}\right\}, x_{3} \in \bar{K}_{R} \backslash\left(K\left(\rho, r_{1}, R\right) \cup \bar{K}_{r}\right) .
$$

\section{Existence theorem}

Theorem 1 Let assumptions $(H 1)-(H 3)$ hold. In addition, we suppose that

(H4) $|c|<\min \left\{\sigma, \frac{d}{D+d}, \frac{\sigma(M-D)}{\sigma(M-D)+D}\right\}$;

(H5) $1-|c|>L \omega(M-d)(1-|c|)+L D \omega|c|$;

(H6) there exist positive constants $r, r_{1}$, and $R$ with $0<r<r_{1}<R$ such that

$$
\begin{aligned}
\frac{\sup _{t \in[0, \omega]} f\left(t, \frac{r}{1-|c|}, \frac{r}{1-|c|}, \frac{C_{0} r}{1-|c|}\right)}{\frac{A_{0} r}{L(1-|c|)}} & <\frac{\sup _{t \in[0, \omega]} f\left(t, \frac{R}{1-|c|}, \frac{R}{1-|c|}, \frac{C_{0} R}{1-|c|}\right)}{\frac{A_{0} R}{L(1-|c|)}} \\
& <\frac{\inf _{t \in[0, \omega]} f\left(t, \frac{\sigma-|c|}{1-c^{2}} r_{1}, \frac{\sigma-|c|}{1-c^{2}} r_{1},-\frac{\sigma-|c|}{\sigma\left(1-c^{2}\right)} C_{0} r_{1}\right)}{\frac{B_{0} r_{1}}{l(1-|c|)}},
\end{aligned}
$$


where

$$
\begin{aligned}
& A_{0}=1-|c|-L \omega(M-d)(1-|c|)-L D \omega|c| \\
& B_{0}=1-|c|-l \omega \sigma(M-D)(1-|c|)+l D \omega|c|
\end{aligned}
$$

Then equation (1) associated with $\lambda \in\left(\lambda_{1}, \lambda_{2}\right)$ has at least three positive $\omega$-periodic solutions, where

$$
\begin{aligned}
& \lambda_{1}=\frac{\frac{B_{0} r_{1}}{l(1-|c|)}}{\bar{b} \omega \inf _{t \in[0, \omega]} f\left(t, \frac{\sigma-|c|}{1-c^{2}} r_{1}, \frac{\sigma-|c|}{1-c^{2}} r_{1},-\frac{\sigma-|c|}{\sigma\left(1-c^{2}\right)} C_{0} r_{1}\right)}, \\
& \lambda_{2}=\frac{\frac{A_{0} R}{L(1-|c|)}}{\bar{b} \omega \sup _{t \in[0, \omega]} f\left(t, \frac{R}{1-|c|}, \frac{R}{1-|c|}, \frac{C_{0} R}{1-|c|}\right)} .
\end{aligned}
$$

Proof By (H5), we obtain $A_{0}>0$ and

$$
\begin{aligned}
B_{0} & >1-|c|-L \omega \sigma(M-D)(1-|c|)-l D \omega|c| \\
& >1-|c|-L \omega \sigma(M-D)(1-|c|)-L D \omega|c| \\
& >1-|c|-L \omega(M-D)(1-|c|)-L D \omega|c| \\
& >1-|c|-L \omega(M-d)(1-|c|)-L D \omega|c| \\
& >0
\end{aligned}
$$

Furthermore, in view of (14), we get $0<\lambda_{1}<\lambda_{2}$.

For each $\lambda \in\left(\lambda_{1}, \lambda_{2}\right)$ and $y \in K$, denote $F$ by

$$
F(y)(t)=f\left(t,\left(A^{-1} y\right)(t),\left(A^{-1} y\right)\left(t-\tau_{1}(t)\right),\left(A^{-1} y\right)^{\prime}\left(t-\tau_{2}(t)\right)\right),
$$

then $F: K \rightarrow C_{\omega}^{+}(\mathbb{R})$ is continuous. We define a mapping $Q_{\lambda}$ by

$$
Q_{\lambda} y=S_{\lambda} \circ F(y)
$$

By Lemma 6, $Q_{\lambda}: K \rightarrow K$ is completely continuous.

Define a function $\rho: K \rightarrow[0, \infty)$ by

$$
\rho(y)=\min _{t \in[0, \omega]} y(t)
$$

Then $\rho$ is a nonnegative continuous concave function on $K$ and

$$
\rho(y) \leq\|y\|_{C}, \quad \forall y \in \bar{K}_{R} .
$$


For any $y \in \bar{K}_{R}$ and $\lambda \in\left(\lambda_{1}, \lambda_{2}\right)$, by (4), (14), (15), Lemmas 4 and 5, we have

$$
\begin{aligned}
\left(Q_{\lambda} y\right)(t) \leq & \int_{t-\omega}^{t} U(t-s)\left[\left(M-a(s) g\left(A^{-1} y\right)(s)\right) y(s)+G(y(s))+\lambda b(s) F(y)(s)\right] d s \\
< & L\left[(M-d) \int_{t-\omega}^{t} y(s)+G(y(s)) d s\right. \\
& \left.+\lambda_{2} \bar{b} \omega \sup _{t \in[0, \omega]} f\left(t, \frac{R}{1-|c|}, \frac{R}{1-|c|}, \frac{C_{0} R}{1-|c|}\right)\right] \\
\leq & L\left[(M-d) \int_{t-\omega}^{t} y(s) d s+\frac{D|c|}{1-|c|} \omega R\right. \\
& \left.+\lambda_{2} \bar{b} \omega \sup _{t \in[0, \omega]} f\left(t, \frac{R}{1-|c|}, \frac{R}{1-|c|}, \frac{C_{0} R}{1-|c|}\right)\right] \\
= & R .
\end{aligned}
$$

Hence $\left\|Q_{\lambda} y\right\|_{C} \leq R$ and $Q_{\lambda}$ is completely continuous on $\bar{K}_{R}$.

We now verify that condition (b) of Lemma 7 holds. Indeed, if $y \in \bar{K}_{r}$, we have

$$
\begin{aligned}
\left(Q_{\lambda} y\right)(t) \leq & L \int_{t-\omega}^{t}\left[\left(M-a(s) g\left(\left(A^{-1} y\right)(s)\right)\right) y(s)+G(y(s))+\lambda b(s) F(y)(s)\right] d s \\
< & L\left[(M-d) \int_{t-\omega}^{t} y(s) d s+\frac{D|c|}{1-|c|} \omega r\right. \\
& \left.+\lambda_{2} \bar{b} \omega \sup _{t \in[0, \omega]} f\left(t, \frac{r}{1-|c|}, \frac{r}{1-|c|}, \frac{C_{0} r}{1-|c|}\right)\right] \\
\leq & L\left[(M-d) \omega r+\frac{D|c|}{1-|c|} \omega r+\frac{A_{0} r}{L(1-|c|)}\right] \\
= & r .
\end{aligned}
$$

Hence, $\left\|Q_{\lambda} y\right\|_{C}<r$.

Choose a positive constant $r_{2}$ such that $0<r_{1}=\sigma r_{2}<r_{2} \leq R$. In the next discussion, we prove that condition (a) of Lemma 7 holds. Obviously, $\rho$ is a concave continuous function on $K$ with $\rho(y) \leq\|y\|_{C}$ for $y \in \bar{K}_{R}$. Noticing that if $y(t)=\frac{1}{3} r_{1}+\frac{2}{3} r_{2}$ for any $t \in[0, \omega]$, then $y \in K\left(\rho, r_{1}, r_{2}\right)$ and $\rho(y)>r_{1}$, which means that $\left\{y \in K\left(\rho, r_{1}, r_{2}\right): \rho(y)>r_{1}\right\} \neq \emptyset$. So, for any $y \in K\left(\rho, r_{1}, r_{2}\right)$, we have

$$
r_{1}<\rho(y)=\min _{t \in[0, \omega]} y(t) \leq\|y\|_{C} \leq r_{2}
$$

Hence, for any $y \in K\left(\rho, r_{1}, r_{2}\right)$, by Lemma 4 , we have

$$
\begin{aligned}
\rho\left(Q_{\lambda} y\right) & =\min _{t \in[0, \omega]} \int_{t-\omega}^{t} U(t-s)\left[\left(M-a(s) g\left(\left(A^{-1} y\right)(s)\right)\right) y(s)-G(y(s))+\lambda b(s) F(y)(s)\right] d s \\
& \geq l \min _{t \in[0, \omega]} \int_{t-\omega}^{t}\left[\left(M-a(s) g\left(\left(A^{-1} y\right)(s)\right)\right) y(s)-G(y(s))+\lambda b(s) F(y)(s)\right] d s \\
& >l\left[(M-D) \int_{t-\omega}^{t} y(s) d s-\frac{D \omega|c|}{1-|c|} r_{1}\right.
\end{aligned}
$$




$$
\begin{aligned}
& \left.+\lambda_{1} \bar{b} \omega \inf _{t \in[0, \omega]} f\left(t, \frac{\sigma-|c|}{1-c^{2}} r_{1}, \frac{\sigma-|c|}{1-c^{2}} r_{1},-\frac{\sigma-|c|}{\sigma\left(1-c^{2}\right)} C_{0} r_{1}\right)\right] \\
\geq & l\left[(M-D) \omega \sigma r_{1}-\frac{D \omega|c|}{1-|c|} r_{1}+\frac{B_{0} r_{1}}{l(1-|c|)}\right] \\
= & r_{1} .
\end{aligned}
$$

Consequently, condition (a) of Lemma 7 holds.

In the end, we prove that condition (c) of Lemma 7 holds.

Let $y \in K\left(\rho, r_{1}, R\right)$ and $\left\|Q_{\lambda} y\right\|_{C}>r_{2}$. We prove $\rho\left(Q_{\lambda} y\right)>r_{1}$. It follows from (15) that

$$
\left\|Q_{\lambda} y\right\|_{C} \leq L \int_{t-\omega}^{t}\left[\left(M-a(s) g\left(\left(A^{-1} y\right)(s)\right)\right) y(s)-G(y(s))+\lambda b(s) F(y)(s)\right] d s .
$$

Therefore,

$$
\begin{aligned}
\rho\left(Q_{\lambda} y\right) & =\min _{t \in[0, \omega]} \int_{t-\omega}^{t} U(t-s)\left[\left(M-a(s) g\left(\left(A^{-1} y\right)(s)\right)\right) y(s)-G(y(s))+\lambda b(s) F(y)(s)\right] d s \\
& \geq l \int_{t-\omega}^{t}\left[\left(M-a(s) g\left(\left(A^{-1} y\right)(s)\right)\right) y(s)-G(y(s))+\lambda b(s) F(y)(s)\right] d s \\
& \geq l \cdot \frac{1}{L}\left\|Q_{\lambda} y\right\|_{C} \\
& >\sigma r_{2} \\
& =r_{1} .
\end{aligned}
$$

Now, all the conditions of Lemma 7 are satisfied. By Lemma $7, Q_{\lambda}$ has at least three positive fixed points $y_{1}, y_{2}$, and $y_{3}$ satisfying

$$
y_{1} \in K_{r}, \quad y_{2} \in\left\{y \in K\left(\rho, r_{1}, R\right): \rho(y)>r_{1}\right\}, \quad y_{3} \in \bar{K}_{R} \backslash\left(K\left(\rho, r_{1}, R\right) \cap \bar{K}_{r}\right) .
$$

Then equation (1) has at least three positive $\omega$-periodic solutions:

$$
x_{1}=A^{-1} y_{1}, \quad x_{2}=A^{-1} y_{2}, \quad x_{3}=A^{-1} y_{3} .
$$

This completes the proof.

Example 1 We consider the positive $2 \pi$-periodic solutions for the second order neutral differential equation

$$
\left(x(t)-0.35 x\left(t-\frac{\pi}{2}\right)\right)^{\prime \prime}+\frac{1}{32} x(t)=\frac{\lambda}{4} F\left(t, x(t), x\left(t-\tau_{1}(t)\right), x^{\prime}\left(t-\tau_{2}(t)\right)\right), \quad t \in \mathbb{R},
$$

where $\lambda>0$ is a constant. Corresponding to equation (1), we choose

$$
c=0.35, \quad \delta=\frac{\pi}{2}, \quad a(t) \equiv \frac{1}{32}, \quad g(x(t)) \equiv 1, \quad b(t) \equiv \frac{1}{4} .
$$


Let $M=\frac{1}{16}$. Then

$$
\begin{aligned}
& \beta=\frac{1}{4}, \quad L=\frac{4}{\sqrt{2}}, \quad L_{1}=\frac{1}{2}, \quad \ell=2, \\
& C_{0}=\frac{1}{4}, \quad \sigma=\frac{\sqrt{2}}{2}, \quad \bar{b}=\frac{1}{4}, \quad D=d=\frac{1}{32} \text {. }
\end{aligned}
$$

Therefore, it is easy to verify that conditions $(H 2)-(H 5)$ are satisfied.

Let $F \in C\left(\mathbb{R} \times[0, \infty)^{2} \times \mathbb{R},[0, \infty)\right), F(t, u, v, w)$ is nondecreasing with respect to $u, v, w$ and $\omega$-periodic with respect to $t$. If there exist positive constants $r, r_{1}$, and $R$ with $0<r<$ $r_{1}<R$ such that the function $F$ satisfies the following inequalities:

$$
\begin{aligned}
\frac{\sup _{t \in[0, \omega]} F\left(t, \frac{r}{1-|c|}, \frac{r}{1-|c|}, \frac{C_{0} r}{1-|c|}\right)}{\frac{A_{0} r}{L(1-|c|)}} & <\frac{\sup _{t \in[0, \omega]} F\left(t, \frac{R}{1-|c|}, \frac{R}{1-|c|}, \frac{C_{0} R}{1-|c|}\right)}{\frac{A_{0} R}{L(1-|c|)}} \\
& <\frac{\inf _{t \in[0, \omega]} F\left(t, \frac{\sigma-|c|}{1-c^{2}} r_{1}, \frac{\sigma-|c|}{1-c^{2}} r_{1},-\frac{\sigma-|c|}{\sigma\left(1-c^{2}\right)} C_{0} r_{1}\right)}{\frac{B_{0} r_{1}}{l(1-|c|)}},
\end{aligned}
$$

where $A_{0} \approx 0.095, B_{0} \approx 0.602$, then equation (16) has at least three positive $2 \pi$-periodic solutions provided that

$$
\frac{\frac{B_{0} r_{1}}{l(1-|c|)}}{\bar{b} \omega \inf _{t \in[0, \omega]} F\left(t, \frac{\sigma-|c|}{1-c^{2}} r_{1}, \frac{\sigma-|c|}{1-c^{2}} r_{1},-\frac{\sigma-|c|}{\sigma\left(1-c^{2}\right)} C_{0} r_{1}\right)}<\lambda<\frac{\frac{A_{0} R}{L(1-|c|)}}{\bar{b} \omega \sup _{t \in[0, \omega]} F\left(t, \frac{R}{1-|c|}, \frac{R}{1-|c|}, \frac{C_{0} R}{1-|c|}\right)}
$$

\section{Acknowledgements}

The authors thank the referees and editors for their careful reading of the manuscript and valuable comments, which helped to improve the quality of the paper.

\section{Funding}

The research is supported by the National Natural Science Function of China (No. 11701457) and Gansu Science and Technology Plan (No. 17JR5RA071).

\section{Availability of data and materials}

Not applicable.

\section{Competing interests}

The authors declare that they have no competing interests in this manuscript

\section{Authors' contributions}

The main idea of this paper was proposed by HY. HY and LZ prepared the manuscript initially and performed all the steps of the proofs in this research. All authors read and approved the final manuscript.

\section{Publisher's Note}

Springer Nature remains neutral with regard to jurisdictional claims in published maps and institutional affiliations.

Received: 27 January 2020 Accepted: 8 April 2020 Published online: 19 April 2020

\section{References}

1. Li, T., Rogovchenko, Y.V.: Oscillation of second-order neutral differential equations. Math. Nachr. 288, 1150-1162 (2015)

2. Jiang, Y., Fu, Y., Wang, H., Li, T.: Oscillation of forced second-order neutral delay differential equations. Adv. Differ. Equ. 2015, Article ID 223 (2015)

3. Li, T., Rogovchenko, Y.V.: Oscillation criteria for second-order superlinear Emden-Fowler neutral differential equations. Monatshefte Math. 184, 489-500 (2017)

4. Chiu, K.S., Li, T.: Oscillatory and periodic solutions of differential equations with piece-wise constant generalized mixed arguments. Math. Nachr. 292, 2153-2164 (2019)

5. Džurina, J., Grace, S.R., Jadlovská, I., Li, T.: Oscillation criteria for second-order Emden-Fowler delay differential equation with a sublinear neutral term. Math. Nachr. (2020). https://doi.org/10.1002/mana.201800196 
6. Li, T., Rogovchenko, Y.V.: On the asymptotic behavior of solutions to a class of third-order nonlinear neutral differential equations. Appl. Math. Lett. 105, Article ID 106293 (2020). https://doi.org/10.1016/j.aml.2020.106293

7. Luo, Y., Wang, W., Shen, J.: Existence of positive periodic solutions for two kinds of neutral functional differential equations. Appl. Math. Lett. 21, 581-587 (2008)

8. Wang, Q., Dai, B.: Three periodic solutions of nonlinear neutral functional differential equations. Nonlinear Anal. 9, 977-984 (2008)

9. Serra, E.: Periodic solutions for some nonlinear differential equations of neutral type. Nonlinear Anal. 17(2), 139-151 (1991)

10. Candan, T.: Existence of positive periodic solutions of first order neutral differential equations with variable coefficients. Appl. Math. Lett. 52, 142-148 (2016)

11. Zhang, M.: Periodic solutions of linear and quasilinear neutral functional differential equations. J. Math. Anal. Appl. 189, 378-392 (1995)

12. Lu, S., Ge, W.: On the existence of periodic solutions for neutral functional differential equation. Nonlinear Anal. 54(7), 1285-1306 (2003)

13. Wu, J., Wang, Z:: Two periodic solutions of second-order neutral functional differential equations. J. Math. Anal. Appl. 329(1), 677-689 (2007)

14. Li, Y.: Positive periodic solutions of second order neutral functional differential equations with delayed derivatives. Acta Math. Sin. 57(3), 505-516 (2014) (in Chinese)

15. Li, Y., Kuang, Y.: Periodic solutions of periodic delay Lotka-Volterra equations and systems. J. Math. Anal. Appl. 255, 260-280 (2001)

16. Leggett, R., Williams, L.: Multiple positive fixed points of nonlinear operators on ordered Banach spaces. Indiana Univ. Math. J. 28(4), 673-688 (1979)

17. Kuang, Y:: Delay Differential Equations with Application in Population Dynamics. Academic Press, London (1993)

\section{Submit your manuscript to a SpringerOpen ${ }^{\circ}$ journal and benefit from:}

- Convenient online submission

- Rigorous peer review

- Open access: articles freely available online

- High visibility within the field

Retaining the copyright to your article

Submit your next manuscript at $\gg$ springeropen.com 